\title{
Peripheral Ulcerative Keratitis following Laser in situ Keratomileusis
}

\author{
Bryn M. Burkholder Irene C. Kuo \\ Wilmer Eye Institute, Department of Ophthalmology, Johns Hopkins University School of \\ Medicine, Baltimore, Md., USA
}

\section{Key Words}

Necrotizing keratitis · In situ keratomileusis · Surgically induced keratitis · Peripheral ulcerative keratitis $\cdot$ Autoimmune disease $\cdot$ Postinfectious complication

\begin{abstract}
Purpose: We report a case of a patient with a history of glomerulonephropathy, not disclosed prior to laser in situ keratomileusis (LASIK), who developed severe postoperative peripheral ulcerative keratitis (PUK) soon after surgery. Method: Case report. Results: Within a week of surgery, the patient, who had no blepharitis or ocular surface disease, also developed diffuse lamellar keratitis (DLK) that was not contiguous with the PUK. Microbiologic evaluation of the flap interface disclosed no organisms, and no epithelial ingrowth was found. Both PUK and DLK resolved with topical and oral steroid therapy, and the patient's induced refractive error improved over the 12 months following LASIK. Conclusions: Necrotizing keratitis has been described after LASIK surgery in patients with or without autoimmune disease. However, to our knowledge, there has been no case of PUK following LASIK. As shown by our patient's clinical course and the typical association of PUK with systemic conditions, patients with a history of atypical postinfectious sequelae may require additional preoperative counseling, vigilant postoperative monitoring, and possibly additional intervention. Because patients do not always divulge medical details, especially if an extraocular site was involved or illness occurred many years prior, this case demonstrates the importance of performing a diligent history that excludes autoimmune disorders or atypical postinfectious sequelae prior to proceeding with keratorefractive intervention.
\end{abstract}


Burkholder and Kuo: Peripheral Ulcerative Keratitis following Laser in situ Keratomileusis

\section{Introduction}

Necrotizing keratitis after ocular surgery is a well-established phenomenon, particularly in patients with a history of autoimmune disease [1-6], although it has been reported in healthy patients as well. In a review of 52 eyes of 43 patients with surgically induced necrotizing sclerokeratitis, $63 \%$ had evidence of an underlying systemic disease, most commonly connective tissue disease [2]. Similarly, sterile peripheral corneal infiltrates can occur after laser in situ keratomileusis (LASIK) in both healthy patients [7-9] and those with a history of systemic autoimmune disease [10-12]. We report a case of bilateral peripheral ulcerative keratitis (PUK) following LASIK in a patient with a history of glomerulonephropathy.

\section{Case Report}

A 29-year-old Filipino woman with contact lens intolerance presented for refractive surgery evaluation. She had been seen elsewhere for episodes of acute red eye and irritation and had been noted to have peripheral corneal infiltrates, attributed to contact lens overwear. She only reported a history of alopecia areata.

Manifest refraction was $-6.00+2.50 \times 090$ in the right eye and $-8.25+3.25 \times 089$ in the left eye, yielding a vision of $20 / 20$ in each eye. Schirmer's testing for dry eye was $>10 \mathrm{~mm}$; she had no rosacea, corneal scars, blepharitis, or ocular surface disease. She underwent uncomplicated femtosecond-assisted (Intralase, Abbott Medical Optics, Santa Ana, Calif., USA) wavefront-guided LASIK using the VISX S4 excimer laser (Abbott Medical Optics). Immediately after surgery, she had extreme photophobia and tearing but no epithelial defect. Moxifloxacin $0.5 \% 3$ times a day, prednisolone acetate $1 \% 6$ times a day, and preservative-free artificial tears 8 times a day were prescribed.

On postoperative day (POD) 1, the patient was extremely photophobic. Uncorrected visual acuity (UCVA) was 20/25 in each eye. Examination revealed stromal opacification within the peripheral right eye flap for $180^{\circ}$, with overlying epithelial defects, but no stromal loss or infiltrate. The left eye exhibited a similar finding for one clock hour outside the hinge. Bandage contact lenses were placed for comfort, and prednisolone acetate was increased to 8 times a day.

Two days later, the left eye developed grade 1 diffuse lamellar keratitis (DLK) not adjacent to the flap findings. Hourly prednisolone acetate and oral prednisone $60 \mathrm{mg}$ were initiated.

Over the next 2 days, the stromal opacification extended annularly in the flaps as well as peripheral to the flaps, similarly with overlying epithelial defects; DLK developed in the right eye; the periphery of both flaps thinned, and visual acuity dropped to 20/100 in the right eye and 20/40 in the left eye. DLK not contiguous with the stromal or flap changes progressed to grade 4 in both eyes.

Both flaps were lifted. The tissue was friable (fig. 1a-c), and no epithelial ingrowth was found. Microbiological cultures and smears were obtained, and the interface was irrigated with moxifloxacin $0.5 \%$ and prednisolone acetate $1 \%$ and replaced. Moxifloxacin and prednisolone acetate drops were increased, and oral prednisone was continued. Bacterial and fungal smears and cultures were negative; the antibiotic drops were tapered, and both topical and oral steroids were continued. On POD 11, visual acuity was 20/50 in the right eye and 20/40 in the left eye with pinhole improvement to 20/25 in each eye. Prednisone and antibiotic drops were stopped on POD 14; topical prednisolone acetate was tapered over 3 
Burkholder and Kuo: Peripheral Ulcerative Keratitis following Laser in situ Keratomileusis

months. The abnormalities in the flap and peripheral corneal stroma gradually resolved. Six months after surgery, UCVA was 20/70 in the right eye (refracting to 20/20 with $-2.50+$ $1.50 \times 150$ ) and $20 / 20$ in the left eye (fig. 1 d, e).

Upon further questioning, the patient revealed that 7 years earlier, she had undergone a renal biopsy for persistent proteinuria following a urinary tract infection; she had not hitherto mentioned the biopsy as she had not considered it a surgical procedure. About a week after LASIK, she was referred to a rheumatologist, who found a left knee effusion. Her complete blood count and metabolic panel, negative antineutrophil cytoplasmic antibodies, rheumatoid factor, cyclic citrullinated peptide, rapid plasma reagin, human leukocyte antigen B27, and erythrocyte sedimentation rate were normal; her antinuclear antibody titer was 1:40. Urinalysis and spot urine protein:creatinine ratio were normal. The rheumatologist concluded that she had no rheumatologic condition.

The patient obtained electron microscopy images of her renal biopsy. The consulting pathologist described an immune complex-mediated process, characterized by electron dense deposits in the subepithelial glomerular basement membrane with mild foot process effacement (fig. 2). The process was most consistent with membranous glomerulonephropathy; postinfectious glomerulonephropathy could not be ruled out.

Because of this history, a nephrology consultation was requested, which the patient scheduled 6 months after LASIK; urine testing, renal function, antinuclear antibody titers, serum protein electrophoresis, and complement levels were unremarkable. The nephrologist concluded that the patient had had a postinfectious glomerulonephropathy that did not warrant follow-up.

Twelve months after LASIK, UCVA was 20/30 in the right eye and 20/20 in the left eye. Manifest refraction of $-1.00+1.00 \times 160$ in the right eye yielded a vision of $20 / 20$; manifest refraction of $-0.75+0.50 \times 175$ in the left eye yielded no further improvement. UCVA, BCVA, and manifest refraction were stable through postoperative month 24 when the patient returned with severe episcleritis in the right eye that responded to topical fluorometholone drops.

\section{Discussion}

We report a case of PUK following LASIK in a patient with a distant history of glomerulonephropathy. To our knowledge, there has been only one reported case of keratolysis in a patient with isolated autoimmune renal disease - a woman with a history of glomerulonephritis who developed scleral necrosis and PUK following cataract extraction [6]. Moreover, to our knowledge, there has been no other case report of PUK following LASIK; the cases of 'necrotic keratitis' in the literature have occurred within the flap or at the flap edge in patients with systemic conditions, like (quiescent) ulcerative colitis [10] and Crohn's disease [11]. We postulate a type of pathergy response in our patient, with trauma inciting robust inflammation. The urinary tract infection may have been the predisposing event for her glomerulonephropathy, and similarly, LASIK may have incited the PUK.

The Food and Drug Administration considers autoimmune disease to be a contraindication to LASIK because of the potential for unpredictable wound healing and keratolysis (http://www.amo-inc.com/products/refractive/ilasik/star-s4-ir-excimer-laser) [13]. Although some surgeons have reported no operative or postoperative complications in patients with controlled or inactive autoimmune disease [13-15], other reports have shown significant postoperative complications in this patient population, including two reported cases of necrotizing keratitis at the flap edge after LASIK in patients with inflammatory bowel dis- 
Burkholder and Kuo: Peripheral Ulcerative Keratitis following Laser in situ Keratomileusis

ease; one patient also had a history of uveitis $[10,11]$. A third case of necrotizing keratitis was described in a patient with well-controlled rheumatoid arthritis with 'faint stromal scarring' on preoperative examination [12]. This patient subsequently developed bilateral corneal infiltrates following unilateral LASIK, suggesting that surgery triggered a global inflammatory process.

A wide variety of systemic diseases have been associated with PUK, including rheumatoid arthritis, Wegener's granulomatosis, relapsing polychondritis, polyarteritis nodosa, microscopic polyangiitis, Churg-Strauss syndrome, and inflammatory bowel disease $[15,16]$. The mechanism of post-LASIK PUK or necrotizing keratitis is not fully understood. Like PUK associated with systemic conditions, the mechanism in post-LASIK eyes may involve local and systemic immunological factors. One key element in PUK is the dysregulation of proteinases and collagenases, produced by inflammatory cells, keratocytes, and the adjacent conjunctiva [16]. Corneal specimens from patients with PUK show an overexpression of matrix metalloproteinases [17], which is also found at higher levels in tear samples of patients with connective tissue disease [18]. The keratolytic pathway may involve corneal deposition of immune complexes, facilitated by limbal vessels that allow rapid recruitment of inflammatory mediators [1]. Other theories include aberrant expression of HLA-2 antigens by keratocytes [1], molecular mimicry between an ocular antigen and a tissue antigen in a remote site [2], and an underlying vasculitic process [4]. The finding of peripheral corneal stromal changes, not just at the flap edge - which can be seen in ocular conditions like epithelial ingrowth as well as in systemic conditions like inflammatory bowel disease - and her return almost 2 years later with episcleritis suggest that the patient may have an underlying systemic condition for which laboratory testing is not conclusive. It is possible that the episodes of acute red eye and irritation she reported prior to LASIK were episcleritis and not contact lens related as originally purported.

Late-onset DLK can be associated with systemic inflammatory conditions ( 9 months to 4 years after LASIK as reported in the literature) [19, 20]; however, in this patient case, DLK occurred within 5 days. It may have occurred with influx of inflammatory cells due to delayed or problematic wound healing at the flap margins [21] or due to the epithelial defects [22]. Although central DLK can lead to stromal tissue degradation, flap melting, and irregular astigmatism, this patient recovered with an UCVA of 20/20 in the left eye and had mild regular astigmatism in the right eye, which accounted for UCVA of 20/30.

Although our patient had no ocular surface disease or established systemic disease at the time of LASIK surgery that would predispose her to PUK or any other sort of necrotizing keratitis, her history of renal dysfunction after a urinary tract infection suggests that she may be predisposed to a more robust inflammatory response (pathergy) to infection, trauma, or surgery. It is possible that the prolonged proteinuria after urinary tract infection and PUK following LASIK were coincidental, but given that each event is a rare complication of a relatively common infection or procedure, such coincidence would seem highly unlikely.

This case underscores the importance of obtaining a thorough medical history prior to LASIK. As illustrated by our case and that of another patient who did not disclose a history of ulcerative colitis because a colectomy had been done [10], patients may not disclose details of inactive disease or understand that the eye can be a secondary site of inflammation. In conclusion, this case demonstrates the importance of performing a diligent history that excludes autoimmune disorders or atypical postinfectious sequelae prior to proceeding with keratorefractive procedures. Patients with a history of autoimmune disease or atypical postinfectious sequelae may require additional counseling prior to keratorefractive surgery, close postoperative monitoring, and additional interventions. 
Burkholder and Kuo: Peripheral Ulcerative Keratitis following Laser in situ Keratomileusis

\section{Acknowledgements}

This work was supported by an unrestricted grant from Research to Prevent Blindness, New York, N.Y., USA.

\section{Statement of Ethics}

This case was in accordance with the principles of the Declaration of Helsinki and was approved by the Institution's Review Board of Johns Hopkins Medicine.

\section{Disclosure Statement}

The authors have no financial disclosures.

\section{References}

1 Maffett MJ, Johns KJ, Parrish CM, Elliott JH, Glick AD, O’Day DM: Sterile corneal ulceration after cataract extraction in patients with collagen vascular disease. Cornea 1990;9:279-285.

-2 O’Donoghue E, Lightman S, Tuft S, Watson P: Surgically induced necrotising sclerokeratitis (SINS) precipitating factors and response to treatment. Br J Ophthalmol 1992;76:17-21.

-3 Perez VL, Azar DT, Foster CS: Sterile corneal melting and necrotizing scleritis after cataract surgery in patients with rheumatoid arthritis and collagen vascular disease. Semin Ophthalmol 2002;17:124-130.

-4 Diaz-Valle D, Benitez del Castillo JM, Castillo A, Sayagues O, Banares A, Garcia-Sanchez J: Immunologic and clinical evaluation of postsurgical necrotizing sclerocorneal ulceration. Cornea 1998;17:371-375.

5 Akpek EK, Demetriades A, Gottsch JD: Peripheral ulcerative keratitis after clear corneal cataract extraction. J Cataract Refract Surg 2000;26:1424-1427.

-6 Bloomfield SE, Becker CG, Christian CL, Nauheim JS: Bilateral necrotising scleritis with marginal corneal ulceration after cataract surgery in a patient with vasculitis. Br J Ophthalmol 1980;64:170-174.

7 Bucci MG, McCormick GJ: Idiopathic peripheral necrotizing keratitis after femtosecond laser in situ keratomileusis. J Cataract Refract Surg 2012;38:544-547.

-8 Yu EY, Rao SK, Cheng AC, Law RW, Leung AT, Lam DS: Bilateral peripheral corneal infiltrates after simultaneous myopic laser in situ keratomileusis. J Cataract Refract Surg 2002;28:891-894.

-9 Haw WW, Manche EE: Sterile peripheral keratitis following laser in situ keratomileusis. J Refract Surg 1999;15:61-63.

10 Carp GI, Verhamme T, Gobbe M, Ayliffe WH, Reinstein DZ: Surgically induced corneal necrotizing keratitis following LASIK in a patient with inflammatory bowel disease. J Cataract Refract Surg 2010;36:1786-1789.

-11 Aman-Ullah M, Gimbel HV, Purba MK, van Westenbrugge JA: Necrotizing keratitis after laser refractive surgery in patients with inactive inflammatory bowel disease. Case Rep Ophthalmol 2012;3:54-60.

-12 Lahners WJ, Hardten DR, Lindstrom RL: Peripheral keratitis following laser in situ keratomileusis. J Refract Surg 2003;19:671-675.

13 Cobo-Soriano R, Beltran J, Baviera J: LASIK outcomes in patients with underlying systemic contraindications: a preliminary study. Ophthalmology 2006;113:1118.e1-e8.

14 Alio JL, Artola A, Belda JI, et al: LASIK in patients with rheumatic diseases: a pilot study. Ophthalmology 2005;112:1948-1954.

15 Smith RJ, Maloney RK: Laser in situ keratomileusis in patients with autoimmune diseases. J Cataract Refract Surg 2006;32:1292-1295.

16 Ladas JG, Mondino BJ: Systemic disorders associated with peripheral corneal ulceration. Curr Opin Ophthalmol 2000;11:468-471.

17 Smith VA, Hoh HB, Easty DL: Role of ocular matrix metalloproteinases in peripheral ulcerative keratitis. Br J Ophthalmol 1999;83:1376-1383.

18 Geerling G, Joussen AM, Daniels JT, Mulholland B, Khaw PT, Dart JK: Matrix metalloproteinases in sterile corneal melts. Ann NY Acad Sci 1999;878:571-574.

19 Diaz-Valle D, Arriola-Villalobos P, Sanchez JM, et al: Late-onset severe diffuse lamellar keratitis associated with uveitis after LASIK in a patient with ankylosing spondylitis. J Refract Surg 2009;25:623-625. 
Case Reports in

Ophthalmology

\begin{tabular}{l|l}
\hline Case Rep Ophthalmol 2016;7:9-15 \\
\hline DOI: 10.1159/000443324 & $\begin{array}{l}\text { c } 2016 \text { The Author(s). Published by S. Karger AG, Basel } \\
\text { www.karger.com/cop }\end{array}$ \\
\hline
\end{tabular}
www.karger.com/cop

Burkholder and Kuo: Peripheral Ulcerative Keratitis following Laser in situ Keratomileusis

20 Javaloy J, Barrera C, Munoz G, et al: Spontaneous bilateral, recurrent, late-onset diffuse lamellar keratitis after LASIK in a patient with Cogan's syndrome. J Refract Surg 2008;24:548-550.

21 Noda-Tsuruya T, Toda I, Asano-Kato N, Hori-Komai Y, Fukumoto T, Tsubota K: Risk factors for development of diffuse lamellar keratitis after laser in situ keratomileusis. J Refract Surg 2004;20:72-75.

22 Johnson JD, Harissi-Dagher M, Pineda R, Yoo S, Azar DT: Diffuse lamellar keratitis: incidence, associations, outcomes, and a new classification system. J Cataract Refract Surg 2001;27:1560-1566.
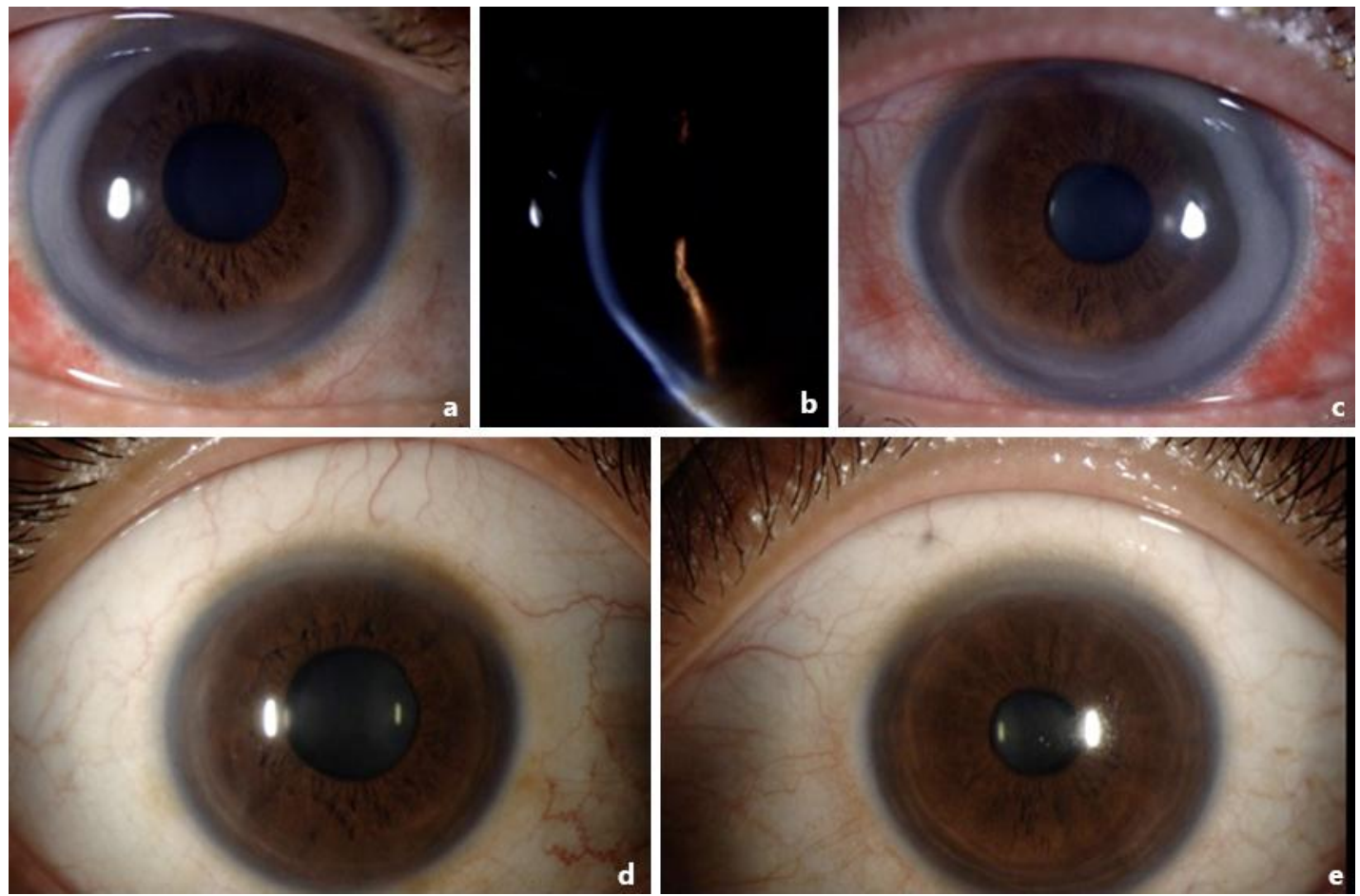

Fig. 1. a-c Slit-lamp photographs on POD 5 (1 day after flap lift and irrigation). a Right eye with improving peripheral keratolysis. b High magnification of peripheral keratolysis in the left eye. $\mathbf{c}$ Left eye with persistent peripheral keratolysis. d, e Photographs at postoperative month 6, showing resolved keratitis with mild peripheral stromal scarring in the right eye (d) and left eye (e). 


\section{Case Reports in \\ Ophthalmology}

Case Rep Ophthalmol 2016;7:9-15

DOI: $10.1159 / 000443324$

(C) 2016 The Author(s). Published by S. Karger AG, Base www.karger.com/cop

Burkholder and Kuo: Peripheral Ulcerative Keratitis following Laser in situ Keratomileusis

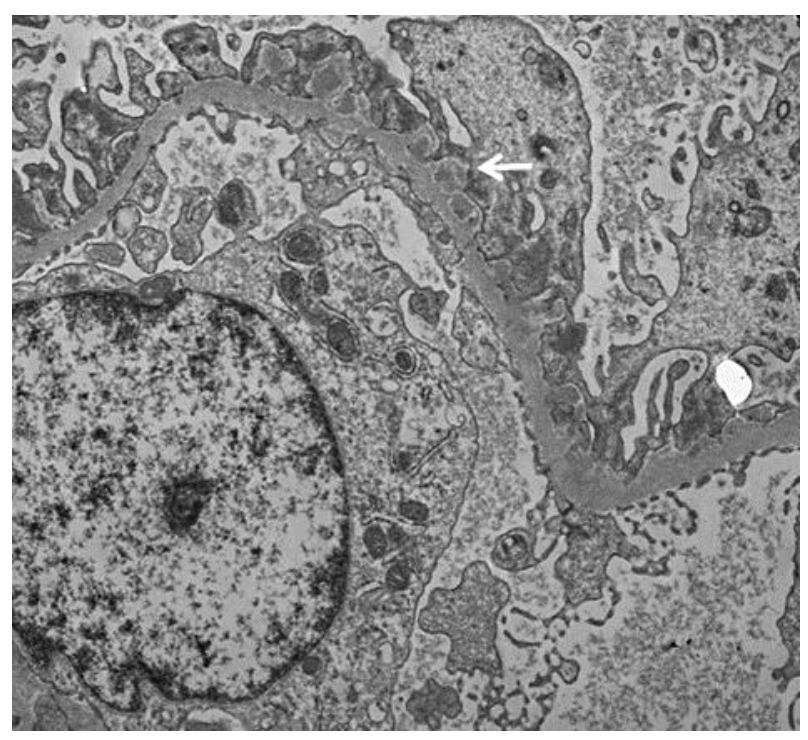

Fig. 2. Electron microscopy of the renal biopsy showing subepithelial membranous deposits (arrow) and adjacent foot process effacement (description courtesy of John Higgins, MD, and Christina Kong, MD, Department of Pathology, Stanford University School of Medicine, Stanford, Calif., USA). 